BULLETIN OF THE

AMERICAN MATHEMATICAL SOCIETY

Volume 81, Number 1, January 1975

\title{
CLOSED ALGEBRAS OF SMOOTH FUNCTIONS
}

\author{
BY CHRISTOPHER I. BYRNES ${ }^{1}$
}

Communicated by I. Kaplansky, May 28, 1974

Introduction. In this note we announce sufficient conditions for an algebra to be a subalgebra of $C^{\infty}(M)$ for some smooth manifold-withoutboundary $M$. In fact, we are able to determine when $M$ is compact and, more generally, when $M$ carries a Riemannian structure. We maintain the notation and terminology used in [5] and [7]. In addition, $m_{p}$ will denote the unique maximal ideal in the stalk $A_{p}$. We assume throughout this note that $A$ is a geometrically homogeneous, harmonic, strongly semisimple, $\mathbf{R}$-algebra with identity. We also assume that $A$ is strongly regular and note that, as a consequence, $\hat{f}$ is a continuous real-valued function on $\Gamma(A)$, for each $f \in A$ [1]. For the sake of brevity, we call an algebra satisfying the above conditions smooth.

Results. If $m_{p}$ is an $A_{p}$-module of finite type, then we set $n$. $\operatorname{dim}_{A}\left(M_{p}\right)$ equal to the minimal number of generators required for $m_{p}$.

DEFINITION 1. If there exists a positive integer $k$ such that for each $M_{p} \in\left(S(A), n \cdot \operatorname{dim}_{A}\left(M_{p}\right)=k\right.$, then we say that $A$ has finite naive dimension $k$, expressed by $n \cdot \operatorname{dim}(A)=k$.

If $\sigma \in H^{0}(U, A)$, then by $[\sigma](p)$ we mean $[\sigma(p)] \in m_{p} / m_{p}^{2}$, where $p \in U$.

Definition 2. $A$ is said to be locally framed if for each $M_{p} \in \mathscr{S}(A)$ there exists a local unit $e_{p}$ at $M_{p}$, a relatively compact open neighborhood $U$ of $p$ with $p \in \bar{U} \subset u\left(e_{p}\right) \subset \Gamma(A)$, and sections $\sigma_{1}, \cdots, \sigma_{k} \in$ $H^{0}(\Gamma(A), A)$ such that the family

$$
\left(\left[\sigma_{1}-\hat{\sigma}_{1}(q) e_{p}\right](q), \cdots,\left[\sigma_{k}-\hat{\sigma}_{k}(q) e_{p}\right](q)\right)
$$

spans $m_{q} / m_{q}^{2}$, where $q \in \bar{U}$ and $k=n \cdot \operatorname{dim}(A)$. The sections $\left.\sigma_{1}\right|_{\bar{U}}, \cdots$, $54 \mathrm{H} 10$.

AMS (MOS) subject classifications (1970). Primary 26A24, 26A93, 46E25, 50A20,

Key words and phrases. Geometrically homogeneous algebra, harmonic algebra, strongly regular algebra, local unit, module of finite type, projective module, derivation, smooth manifold, Riemannian metric.

1 The author's graduate study is currently supported by an NSF Traineeship. 
$\left.\sigma_{k}\right|_{\bar{U}} \in H^{0}(\bar{U}, A)$ will be called a system of parameters for $\bar{U}$.

The problem of deciding when $\Gamma(A)$ is a topological manifold is a local problem, as the following theorem demonstrates.

THEOREM 1. If $U$ is a relatively compact open subset of $\Gamma(A)$, then $\max \left(H^{0}(\bar{U}, A)\right) \cong \Gamma\left(H^{0}(\bar{U}, A)\right) \cong \bar{U}$.

If $\sigma_{1}, \cdots, \sigma_{k}$ is a system of parameters for $\bar{U}$ then for each $i=$ $1, \cdots, k$ we may consider the ideal $I_{i}=\left(\sigma_{1}, \cdots, \sigma_{i-1}, \sigma_{i+1}, \cdots, \sigma_{k}\right)$ in $H^{0}(\bar{U}, A)$.

Definition 3. If $p \in U$ then we say that $A$ is linearly idemfree at $p$ if the algebra $H^{0}(\bar{U}, A) / I_{i}$ has no nontrivial idempotents for $i=1, \cdots$, $k$. If $A$ is linearly idemfree at each $p \in \Gamma(A)$, then we call $A$ linearly idemfree.

THEOREM 2. If $A$ is a smooth, locally framed, linearly idemfree algebra, then $\Gamma(A)$ is a $k$-dimensional topological manifold-without-boundary.

We denote the $A$-module of $\mathbf{R}$-algebra derivations of $A$ by $\operatorname{Der}_{\mathrm{R}}(A)$.

Definition 4. If $A$ is a smooth algebra with $n \cdot \operatorname{dim}(A)=k$, then we say $\operatorname{Der}_{\mathrm{R}}(A)$ is tangent to $A$ provided

(i) $\operatorname{Der}_{\mathrm{R}}(A)$ is a finitely generated projective $A$-module,

(ii) for each $M_{p} \in\left(S(A), \operatorname{Der}_{\mathrm{R}}(A) / M_{p} \operatorname{Der}_{\mathrm{R}}(A)\right.$ is a real vector space of dimension $k$.

Some technical modifications of the methods used in [2] and [6] allow us to linearize the problem of local coordinization, provided $\operatorname{Der}_{\mathbf{R}}(A)$ is tangent to $A$. In fact, this linear approximation is quite adequate.

THEOREM 3. If $A$ is a smooth algebra with $n \cdot \operatorname{dim}(A)=k$ such that $\operatorname{Der}_{\mathbf{R}}(A)$ is tangent to $A$, then $A$ is locally framed.

Definition 5. $A$ is said to be a $k$-differentiable algebra provided

(i) $A$ is smooth,

(ii) $n \cdot \operatorname{dim}(A)=k$,

(iii) $\operatorname{Der}_{\mathbf{R}}(A)$ is tangent to $A$,

(iv) $A$ is linearly idemfree.

Note that if $A$ is a $k$-differentiable algebra then, by Theorems 2 and 3, $\Gamma(A)$ is a topological $k$-manifold. Actually, using a local version of a lemma due to Banaschewski [3], we are able to prove 
THEOREM 4. If $A$ is a $k$-differentiable algebra then $\Gamma(A)$ is a smooth manifold.

DEFINITION 6. If $M$ is a smooth manifold then a harmonic subalgebra $A$ of $C^{\infty}(M)$ is said to be a closed algebra of smooth functions on $M$ provided $A$ contains an atlas for $M$ and $A$ is closed under the differential operators corresponding to this atlas.

Definition 6 enables us to sharpen Theorem 4.

THEOREM 5. If $A$ is a $k$-differentiable algebra then $A$ is isomorphic to a closed algebra of smooth functions on $\Gamma(A)$.

THEOREM 6. If $A$ is a $k$-differentiable algebra with $(S(A)=\operatorname{Max}(A)$, then $A$ is isomorphic to a closed algebra of smooth functions on the compact manifold $\Gamma(A)$.

Bkouche has obtained [4] an algebraic condition which determines when an open subset of $\max (A)$ is paracompact, for certain commutative rings $A$. Using this characterization and the well-known fact that every paracompact smooth manifold admits a Riemannian metric we deduce

THEOREM 7. If $A$ is a $k$-differentiable algebra such that $A_{0}$ is a projective ideal, then $A$ is isomorphic to a closed algebra of smooth functions on the Riemannian manifold $\Gamma(A)$.

Of course, if $M$ is a Riemannian $k$-manifold, then $\mathcal{C}^{\infty}(M)$ is a $k$-differentiable algebra having $C_{0}^{\infty}(M)$, the smooth functions with compact support, as a projective ideal. A more comprehensive treatment of these results will appear elsewhere.

ACKNOWLEDGEMENTS. The author is proud to thank his thesis advisors, Professors N. E. Hurt and M. H. Stone, for the inspiration and guidance he has received from them and their work.

\section{REFERENCES}

1. F. W. Anderson and R. L. Blair, Characterizations of the algebra of all realvalued continuous functions on a completely regular space, Illinois J. Math. 3 (1959), 121-133. MR 20 \#7214.

2. M. F. Atiyah, K-theory, Benjamin, New York, 1967. MR 36 \#7130.

3. B. Banaschewski, An algebraic characterization of $C^{\infty}\left(R^{n}\right)$, Bull. Acad. Polon. Sci. Sér. Sci. Math. Astronom. Phys. 16 (1968), 169-174. MR 37 \#589.

4. R. Bkouche, Pureté, mollesse, et paracompacité, C. R. Acad. Sci. Paris Sér. A-B 270 (1970), A1653-A1655. MR 44 \#4012.

5. C. I. Byrnes, A spectral decomposition theorem for certain harmonic.algebras, Bull. Amer. Math. Soc. 80 (1974), 1271-1275. 
6. R. G. Swan, Vector bundles and projective modules, Trans. Amer. Math. Soc. 105 (1962), 264-277. MR 26 \#85.

7. S. Teleman, Theory of harmonic algebras with applications to von Neumann algebras and the cohomology of locally compact spaces, Lecture Notes in Math., vol. 248, Springer-Verlag, Berlin and New York, 1971.

DEPARTMENT OF MATHEMATICS AND STATISTICS, UNIVERSITY OF MASSACHUSETTS, AMHERST, MASSACHUSETTS 01002 